\title{
Is the Conduct of Medical Research on Chimpanzees Compatible with Their Rights as a Near-Human Species?
}

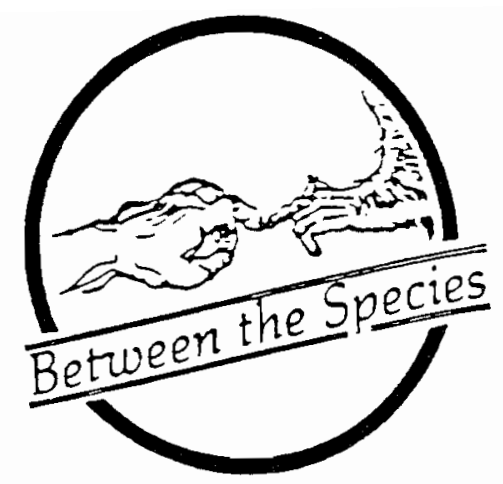

Alfred M. Prince, M.D.

The Liberian Institute for Biomedical Research

Robertsfield, Liberia

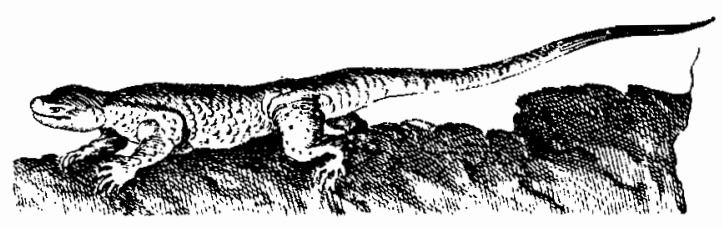

Many animal rights activists consider that all research carried out with animals is indefensible. This would apply especially to research with chimpanzees. I assume that chimpanzees are the closest relatives to humans and that they deserve ethical considerations which are similar to those accorded humans. Nevertheless, I believe that it is ethically justifiable to carry out certain types of experimentation with this species, as it is also with humans. I welcome the opportunity to defend this position here.

My laboratory, Vilab II, has been carrying out research with chimpanzees in Liberia since 1975 . The research, none of which affects the health or well-being of the animals, is directed toward the development of vaccines for prevention of three major human diseases: hepatitis B, hepatitis C, and Onchocerciasis (River Blindness). Chimpanzees are the only nonhuman animal species susceptible to these infections.

We originally acquired chimpanzees by humane capture using anaesthetic darts in a recently logged forest destined for agricultural exploitation. The animals in this region were thus severely threatened, since Liberian farmers do not hesitate to shoot chimpanzees which forage on their crops. Later, we acquired animals who had been held in Liberian or expatriate families as pets, and whose owners could no longer care for them as they emerged from the "cute" and easy to handle juvenile stage. These animals had no future, since humane facilities for housing and care of adult chimpanzees are not available in Liberia, except at Vilab II. Recently, colony born animals have more than satisfied our requirements for additional animals.

Because of our awareness of the near human needs and nature of chimpanzees, a major emphasis of our laboratory has been to maintain animals under conditions which satisfy their physical and emotional needs to a maximal extent. Our approaches to this have been described in detail $(1,2,3)$. Briefly, this has involved our requirement that animals never be housed alone, that they be housed in large outdoor cages from which they can hear and observe many other animals, that foraging type enrichment devices be provided, and finally that after studies are completed, the animals are socialized into progressively larger groups leading ultimately to the release of groups of 20-30 animals on to 12-30 acre islands in nearby rivers where they are maintained in a free living state with moderate food supplementation. The success of our approach was shown by the extraordinary fertility of the released animals. Of 90 animals released on 5 islands, all females of breeding age were either pregnant or carrying babies in 1990 .

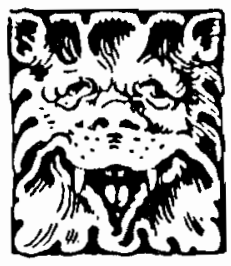


An endowment fund has been established which we hope will ultimately assure the indefinite survival of the island groups. These groups will provide opportunities for behavioral studies, and if safe and secure national parks become established in the region, they could provide a source of animals for restocking of wild chimpanzee populations.

Has the Vilab II experience been ethically defensible? I believe that it has. First, the animals acquired had little or no future, and now have a safe and assured life in which they can live out their lives under natural conditions. Second, their contribution to research has been an essential one: vaccines have been developed with their help for hepatitis B, and are in the process of being developed for hepatitis $C$ and Onchocerciasis, diseases which cause untold human suffering. The experiments carried out with chimpanzees involve challenging putatively immunized animals with live virus or infective larvae. This cannot be done with humans because it entails a risk of death or serious illness. Fortunately, the immune systems of chimpanzees are more effective than ours, and as a result, they have never developed overt illness as a result of these infections. Thus, these important studies can be humanely done in the chimpanzee model. Furthermore, this can be done under conditions which benefit the animals and provide them with a secure and humane future.

If chimpanzees are to be afforded the same ethical considerations as are humans, then it can be legitimate to ask whether similar research could be ethically done with humans. Consider the following imaginary scenario: During a famine in the Sahel, an epidemic of meningococcal meningitis is decimating the starving children of the region. A medical research team wishes to evaluate vaccines which might halt this and other similar epidemics. The studies have to be done in children, since adults are already immune as a result of past epidemics. They therefore set up camps in which orphaned children can be well housed and fed and in which the vaccines can be tested. Because of the age and limited education of the children, obtaining meaningful informed consent is not feasible. Without this project, these children will be in serious danger of starving to death. If illness occurs during the vaccine trials, prompt treatment will be available to avert serious sequelae. It is planned that after the completion of the trials, the children will be returned to their villages in good health, and with sufficient support to provide for their subsistence and an elementary education.

Is the above an ethical project? Are these children being "used"? They are. However this is providing benefit both to the subjects of the trials and to society as a whole, and without unacceptable risk to the subjects themselves. I believe that this imaginary scenario describes an ethically defensible medical research project. However, I recognize that this conclusion, and the project itself, would be controversial. I think that this imaginary project shares essential similarities with the Vilab II chimpanzee research project, and that both can be justified on similar grounds.

\section{References}

1. Prince, A.M: "The use of chimpanzees in biomedical research." Proceedings of the Symposium on Trends in Bioassay Methodology: In Vivo, In Vitro and Mathematical Approaches, February 18-20, 1981, Washington, D.C., (NIH Publication No. 82-2382), pp. 81-97.

2. Prince, A.M.; Goodall, J.; Brotman, B.: Dienske, H.; Schellekens, H.; Eichberg, J.W. "Appropriate conditions for maintenance of chimpanzees in studies with bloodborne viruses: An epidemiologic and psychosocial perspective." J. Med. Primatol. 18:27-42, 1989.

3. Prince, A.M.; Brotman, B.; Garnham, B.; Hannah, A.C. "Enrichment, rehabilitation, and release of chimpanzees used in biomedical research." Lab Animal 19(5):29-37, 1990.

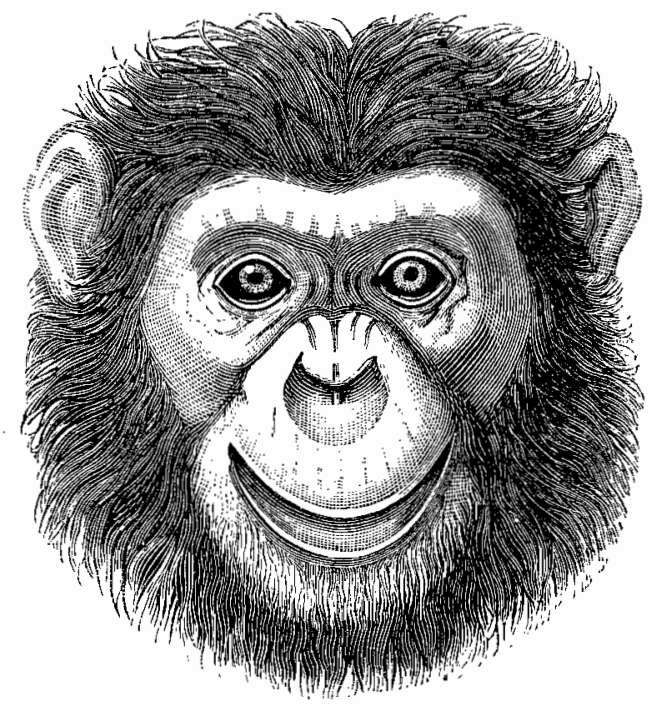

